\title{
SACCADIC OMISSION: WHY WE DO NOT SEE A GREY-OUT DURING A SACCADIC EYE MOVEMENT
}

\author{
Fergus W. Campbell and Robert H. Wurtz
}

The Physiological Laboratory. University of Cambridge. Cambridge CB2 3EG. England: and The National Institute of Mental Health, Bethesda. Maryland 20014. U.S.A.

(Received 20 June 1977; in revised form 4 January 1978)

\begin{abstract}
We investigated why we have no perception of a smeared image resulting from the reduction in contrast (grey-out) occurring at the retina during saccadic eye movements. By turning on light in the experimental room only during the eye movement, we were able to show that this grey-out was perceived as a smeared image of the visual scene. However, when the experimental room was illuminated before and/or after the saccade as well as during the saccade, perception of the grey-out was obliterated. During a period of fixation. perception of a blank image comparable in duration to an eye movement could also be etiminated by a preceding or following clear image. We conclude that lack of perception during saccadic eye movements made in normal contoured environments results primarily from the visual "masking" effect of a clear image before and/or aiter the eye movement acting on the grey-out during the eye movement. This "saccadic omission" is entirely a visual phenomenon and is far more powerful than the usually studied elevation of visual threshold for detection of a flash. "saccadic suppression."
\end{abstract}

\section{INTRODUCTION}

Our vision is interrupted several times a second by rapid eye movements (saccades) made from one point in the visual field to another. During these saccades the visual world sweeps actoss the retina and should produce a period of smeared vision lasting from 25 to about $100 \mathrm{msec}$ depending on the size of the saccade (Robinson, 1964; Westheimer, 1954). Yet we normally do not see this smeared image of the visual scene generated by the eye movement.

This lack of perception is easily demonstrated by attempting to see one's own eye movements in a mirror, as described by Dodge (1900): "It was then we chanced on the observation that when the head was held perfectly still we could never catch our own eyes moving in a mirror. One may watch one's eyes as closely as possible, even with the aid of a concave reflector, whether one looks from one eye to the other, or from some more distant object to one's own eyes, the eyes may be seen now in one position and now in another, but never in motion." If one looks at a colleague's eyes in the mirror, one can easily see his eye movements.

Most of the work since Dodge $(1900,1905)$ and his contemporaries has concentrated on eliminating the smeared image occurring during a saccade in order to reveal the existence of any central inhibition associated with the eye movement. Attempts to quantify any such effect by testing the elevation of threshold for detecting a brief flash of light during a saccadic eye movement have generally found rather small effects, usually less than $0.5 \mathrm{log}$ units (see review by Matin, 1974). This elevation of threshold has been named "saccadic suppression" (as cited by Zuber and Stark, 1966) but would be more accurately described as saccadic attenuation, and it is certainly not adequate to explain our lack of vision during an eye movement.

In addition, we think these experiments on saccadic suppression fail to deal with a central problem of vision during eye movements: Why are we unaware of the physical event occurring at the retina-a greyout which should result in perception of a smeared visual scene? By grey-out we mean a decrease in the contrast of an image due to its movement across a retina which integrates over time. This is analogous to the image on photographic film when the camera is moved over a wide angle while the shutter is open. The problem is thus not one of an elevation of threshold or reduction in sensitivity during eye movements-a saccadic suppression-but an elimination of this perception which we will refer to as a saccadic omission.

The present experiments demonstrate that this saccadic omission can result from the presence of a clear image before and/or after a saccade acting on the grey-out during a saccade. Such "masking" effects have been suggested previously, and experimentally demonstrated by Matin, Clymer and Matin (1972). From our experiments we conclude that in a contoured visual environment the "masking" of the greyout by the clear image is the primary factor eliminating perception during saccadic eye movements. that is, in producing the saccadic omission.

\section{METHODS}

We made it possible to light the visual scene in front of a subject only during an eye movement. The light source used was a repetitive xenon flash which enabled us to turn on and off a bright light within several msec of a trigger pulse. The flash tube was run at such a rate that it was perceived as a steady light. We adjusted the intensity of the light so that a $100 \mathrm{msec}$ period of flashes was seen as having a luminance of $3 \mathrm{~cd} / \mathrm{m}^{2}$.

The subject sat in a darkened room and made saccades from one small red fixation light (from a light-emitting diode) to another placed $20^{\circ}$ or $30^{\circ}$ away in the horizontal plane. This configuration of fixation lights produced eye movements that were stable in duration and time-course as long as the subject remained alert. 
Horizontal eye movements were recorded using electrooculograms (EOG) derived from two silver-silver chloride electrodes (Beckman. Miniature Biopotential) pasted to the cleaned skin at the outer canthus of each eye. This signal was displayed on a storage oscilloscope and also differentiated for use as a trigger for turning on the light during the saccades. The duration of any period of light and the frequency of the component flashes were recorded by a silicon photocell in front of the flash tube and were dis. played on the second channel of the storage oscilloscope. The temporal relations between the saccadic eye movement and the stimulus were determined from the storage oscilloscope for each trial.

The light stumulus. consisting of the repetitive flashes at $1 \mathrm{kHz}$, was usually set to fill the duration of the saccade. To obtain a period of light after the end of the eye movement the duration of the triggered light stimulus was simply extended by up to $200 \mathrm{msec}$ beyond the end of the saccade. A period of light just before the saccade was obtained by adding, to the constant pertod of light flling the duration of the saccade. another period of light synchronized with the onset of one of the fixation lights. This pertod of light came on after a delay to allow for the reaction time of the subject. Only those trials were used in which the reaction tume placed the light period just before the eye movement.

A further set of experiments used a sequence of stimuli whle the subject fixated. In these experiments the successive stimuli were from two xenon flash tubes each flashing at $320 \mathrm{~Hz}$. The flash tubes were used as the light source for two slide projectors whose beams were combined using a beam-splitter and then back-projected onto a groundglass screen. The apparent luminance was adjusted to be $3 \mathrm{~cd} \mathrm{~m}^{2}$. and matching of slight color differences between the tubes was aided by inserting a yellow (minus blue) filter in the beam.

\section{RESULTS}

\section{Grey-out due to eye motement}

We first confirmed that it was possible to see clearly during an eye movement. In a dark room a subject fxated a light on the left; a second light then came on $20^{\circ}$ to the right and the subject made a saccade to it. This eye movement took $50-70 \mathrm{msec}$ after the subject's reaction time (see Fig. 1, insert). The EOG of the eye movement triggered the repetitive xenon flashes illuminating the scene before the subject. In this initial experiment the scene consisted largely of the usual bank of oscilloscopes, oscillators and recording apparatus found in the laboratory. Highlights, such as very reflective parts, were covered with black tape. As the subject began a saccade, the light in the room was turned on within about $10 \mathrm{msec}$ after onset of the eye movement, and left on for a duration of $5 \mathrm{msec}$ or less (1-5 flashes). The room was seen clearly no matter where in the $50-70 \mathrm{msec}$ eye movement the period of light occurred. This, of course, is not surprising since the light was brief enough to minimize any smearing of the image across the photoreceptors of the retina.

We then turned on the light and left it on for the $50-70 \mathrm{msec}$ duration of the saccade. After each eye movement, the subject was asked whether the scene appeared as smeared or sharp. In every instance the subject said promptly that the scene was smeared, that is, it was greyed-out and appeared strikingly different from the clarity produced during the short exposure described above. This smeared appearance

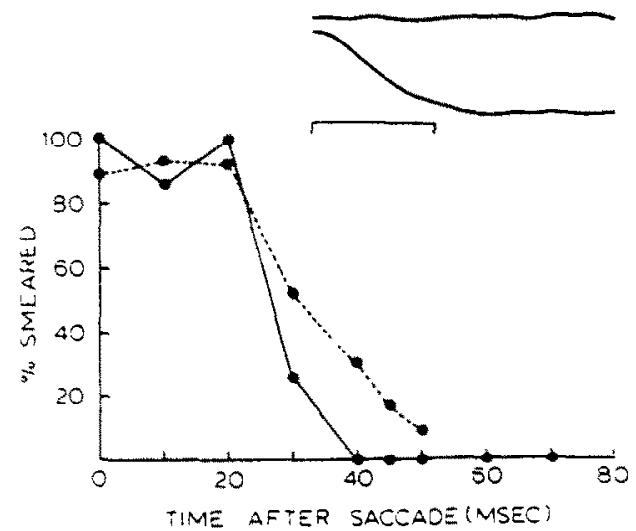

Fig. 1. Transition of smeared to clear image as the period of light extends beyond the end of the saccade. A $50 \mathrm{msec}$ period of light was on during the eye movement on every trial. Ordinate shows percent of time the subject (FWC) reported that the image was smeared. The abscissa shows the duration of the light after the end of the eye movement. Circles connected by solid lines show the percent of the time the subject saw the scene as smeared as he looked around the laboratory room. (Circles connected by dashed lines show percent of time the subject saw a smeared image of his moving eye as he looked at his eyes in a mirror.) Inset shows an oscilloscope sweep of the EOG of a saccade used to trigger a $50 \mathrm{msec}$ period of light during the eve movement indicated by the bracket. Line above saccade shows EOG level before the saccade.

is what would be expected physically as the retina sweeps over the scene at a peak velocity of at least $500-600 \%$ sec.

As we extended the duration of the light beyond the end of the eye movement, the amount of smearing that the subject reported became progressively less. The permitted responses were smeared, slightly smeared, and sharp. These responses were assigned values on a linear rating scale and are summarized as percent smeared in Fig. 1. The circles connected by solid lines in Fig. I show the percentage of the time that the scene was reported as smeared against the time the light was left on beyond the end of the eye movement. The end of the eye movement was determined on each trial by inspecting the trace of the EOG on the storage oscilloscope. This allowed for the slight variability in the duration of each saccade (the error in determining the end of the saccade was about $\pm 5 \mathrm{msec}$ ). At least three trials in random order were made for each duration of light shown on the abscissa of Fig. 1.

By the time the light extended $40 \mathrm{msec}$ beyond the end of the eye movement the subject reported a clear scene $100 \%$ of the time. It is important to note that the subject did not perceive a smeared image followed by a sharp image but instead reported only a single percept even though the $50 \mathrm{msec}$ of grey-out was still physically present. Furthermore, he was not aware that the duration of the light varied in different trials until the light extended more than $80-100 \mathrm{msec}$ beyond the end of the eye movement.

A second subject reported essentially the same absence of smearing when the light extended beyond the end of the eye movement. The same effect was observed with saccades $5^{\circ}, 10^{\circ}$ and $20^{\circ}$ long. We also repeated the same experiment with glow-modulator 


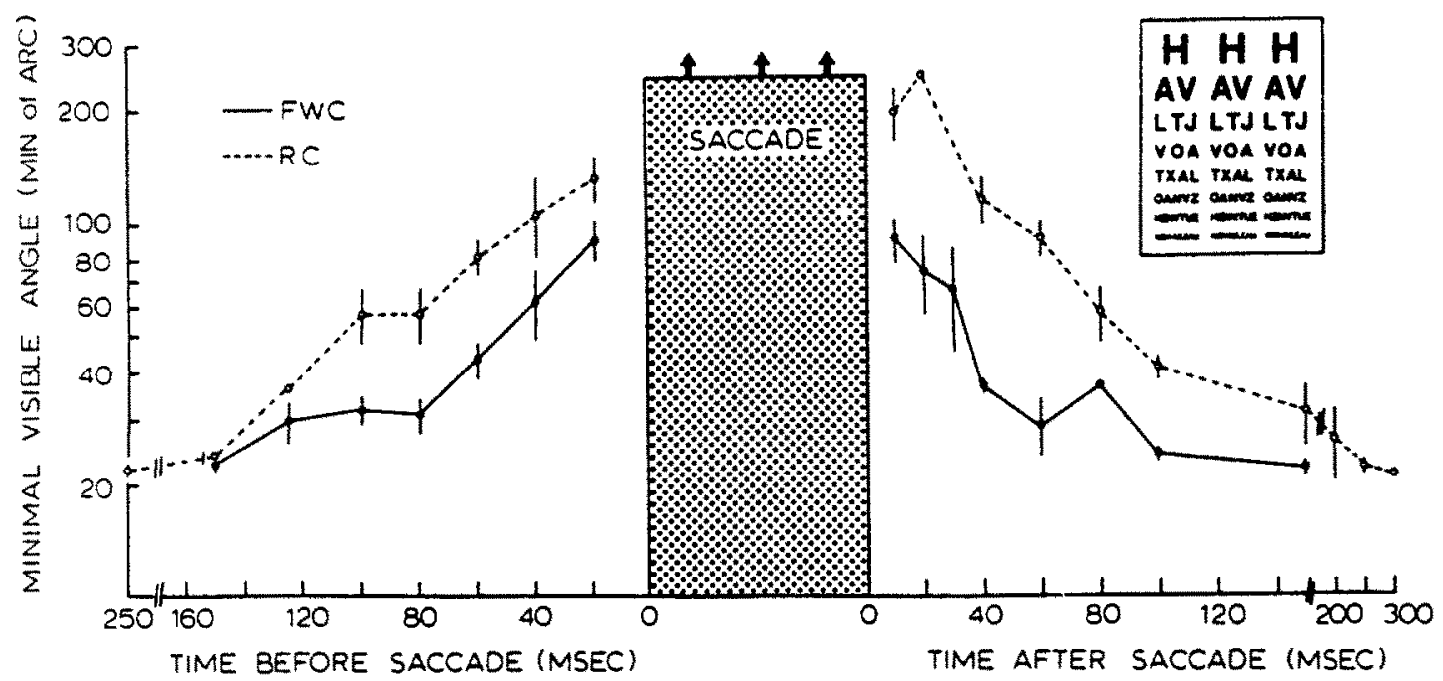

Fig. 2. Effect of a clear image before and after the smeared image during a saccade. The ordinate shows the minimal visible angle seen on the Snellen test charts: a logarithmic scale is used. Abscissa shows the time the period of light extended before or after the eye movement. Vertical line at each point is the standard error: where no line is shown the standard error is smaller than the symbol used. The stippled area indicates the period of light during a $30^{\circ}$ saccade. and upward pointing artows above stippled area indicate that not even largest visible angle on the chart could be seen during the saccade.

tubes instead of the repetitive xenon flashes. We could only illuminate a small portion of the visual scene due to the low light output of the glow modulator tubes but the results obtained were the same: a smeared percept when the light was on only during the eye movement and a clear image when the light extended $50-100 \mathrm{msec}$ beyond the end of the eye movement. From these observations, we conclude that the sharp static image on the retina after the eye movement obscures the presence of the grey-out produced by the eye movement.

We then returned to the original observation that one does not see one's own eyes move when they are viewed in a mirror. Each author viewed his own eyes in a mirror at a distance of $20 \mathrm{~cm}$ (although the distance was not critical) when the eyes were illuminated only during the eye movement. as in the above experiment. Now during the eye movement the black pupil and dark iris were seen as an elongated blurred patch, and the bright comeal reflection was seen as a shorter bright streak. The effect of increasing the duration of the light beyond the end of the saccade as the eye movement was viewed in the mirror is shown by the circles connected by a dashed line in Fig. 1. As the duration of the light increased, the frequency of seeing the blur declined in close agreement with the preceding experiment. Again, in this case only one percept was seen with long light durations; the subject was not aware of the grey-out during the saccade which preceded the sharp image seen during fixation.

These experiments establish that the presence of a static and therefore clear image of a scene obscures the presence of a preceding grey-out even when the grey-out lasts as long as $50-70 \mathrm{msec}$. This raises the question of whether a clear image before the eye movement has a similar effect.

\section{Pre- and post-saccade effects}

In order to quantify the degree of smear more objectively. we measured visual acuity during and after saccades. Our rationale was that the greater the effect of the grey-out. the lower the visual acuity should be just before or just after the eye movement.

The subject made a $30^{\circ}$ saccade between two fixation lights. Three copies of a conventional Snellen test chart were used to fill the space between the fixa. tion lights (as illustrated in the inset of Fig. 2). The subject could raise and lower the chart so that any line he chose was on the level of the fixation lights. As the chart was viewed from a distance of $1.2 \mathrm{~m}$ rather than the standard $6 \mathrm{~m}$ the angle subtended by the letters was five times larger than conventional use. Therefore. the range of acuity covered varied from $21^{\prime}-250^{\prime}$.

Figure 2 shows the results of these experiments for two subjects. The ordinate is a logarithmic scale of the visual acuity in minutes of arc. The abscissa is duration of the light in msec plotted on a linear scale. On the right is the effect of light durations obtained by extending the light after the end of the saccade; on the left is the effect of durations of light before the start of the saccade. The blank section between the two scales represents the duration of the light during the eye movements which was set at $70 \mathrm{msec}$ for subject FWC and $80 \mathrm{msec}$ for subject RC. The data for the periods before and after the eye movements were collected in separate sessions. In each session the order in which different durations of light were presented was randomized. Each point represents the average of three judgements. Each judge. ment was the final result of at least ten presentations of the stimulus which allowed the subject enough trials to move the test chart up and down in order to determine the lowest line which could be read. 
As uas found when vewing the room in the same conditions. the perceived image was so smeared that even the large letters subtending 250' were not readable when the light was on only during the eye movement. (The vertical arrows durng the period of the eye movement indicate that the data cannot be plotted on the scale.)

On the right hand of Fig. 2 the graph shows that the subject can read smaller and smaller letters on the Snellen chart as the duration of the light extends for longer periods beyond the end of the saccade. An exposure of as little as $100 \mathrm{msec}$ after the eye movement produced nearly maximal acuity for subject FWC at the light levels used in this experiment. ${ }^{\prime}$ The duration of the light after the eye movement did not in itself affect acuity: brief duration still permitted the bottom line of the test chart to be read.

On the left of Fig. 2 the results show that if the light was turned on before the eye movement, the longer the light was on, the lower on the chart the lines could be read. Again, visual acuity reached nearly maximal levels when the light was on about $100 \mathrm{msec}$ before the saccade. Although there are some differences between the shape of the curves obtained with light before and after the eye movement. the results show that light both before and after the eye movement is effective in obscuring the smeared image. ' It is important again to emphasize that there was only one percept and that the subjects did not report a blurred percept followed or preceded by a clear one for any of our exposure times, short or long.

We conclude from these experiments that a clear static image either immediately before or after the eye movement obscures the grey-out occurring during the eye movement. although this grey-out is still physically present.

We then determined whether a period of grey-out would be acted on by a clear image in the absence of any eye movements. To do this we projected a blank white scene for $50 \mathrm{msec}$ onto a screen while the subject fixated on the screen and preceded or followed this by a clear image of a Snellen test chart. As in the case of eye movements, maximum acuity was attained when a period of $50-100 \mathrm{msec}$ of the clear image either preceded or followed the blank period. Since the clear image obscures the blank image in the absence of large eye movements, the effect of a clear image on a blank or a grey-out is not dependent on the occurrence of the eye movement but rather is a characteristic of the visual system.

'Differences in the slope of the curves of the two subjects are probably due to differences in accommodation. Subject FWC was a presbyopic uncorrected myope who viewed the screen at his uncorrected far-point. However. $\mathrm{RC}$ was a young corrected myope who focused for a distance beyond the screen. Due to the short duration of the flash there was insufficient time for her to accommodate for the distance of the screen. This point was overlooked at the time of the experiment but was checked later and found to account for her lower overall acuity.

2 The judgment before the eye movement was more difficult to make since it could not be made on successive trials if early or late occurring saccades produced an unusual duration of light preceding the eye-movement. The exact shape of the curve. therefore, may not be significant.
The characteristics of this nsual interaction are considered in detal in the following paper (Corfield. Frosdick and Campbell. 1978).

\section{DISCLSSIOV}

Our experiments have shown that we aluays see a smeared image during an eye movement if illumination of the environment is limited to the duration of the eye movement. However, the presence of a clear image before or after the eye movement eliminates perception of the grey-out even though it is still present at the retina. The shift from perception of a smeared image to a clear image can be made simply by turning a knob controlling the relative duration of clear and smeared images. We therefore suggest that in a normal contoured environment the primary mechanism for eliminating the smeared image must be this visual masking effect with central factors having negligible infuence.

This effect can also be demonstrated independently of saccadic eye movements when a clear image preceds or follows a $50 \mathrm{msec}$ blank image during a period of fixation. The effect of a clear image on a blank or smeared one is thus a functional characteristic of the visual system and is not dependent on the physical motion of the eyeball or its accompanying neuromuscular activity. We will discuss these observations in relation to saccadic suppression and corollary discharge, visual masking and possible physiological correlates.

\section{Saccadic suppression and corollary discharge}

Helmholtz (1910) was probably the first to suggest that a central mechanism operates during an eye movement. He suggested that the stability of the visual world in spite of frequent eye movements was dependent upon a discharge from the motor system to the sensory system. and that this input allowed the sensory system to compensate for the shifted image. This motor-related discharge, which has subsequently been referred to as a corollary discharge (Sperry, 1950) or an efference copy (von Holst. 1954) has also been suggested as a way of elminating vision during saccadic eye movements. Holt (1903) also proposed a "central anaesthesia" initiated by movement of the eye muscles to account for this lack of vision. Our experiments rule out any corollary discharge or central anaesthesia as the primary mechanism for eliminating vision during eye movements for the following reasons: First, we have demonstrated that the smeared retinal image can be seen during an eye movement when the light is on only during a saccade and, of course brief stimuli falling on the retina are seen clearly. A potent corollary discharge should eliminate any such perception. Second. this perception of the smear can be eliminated by simply extending the duration of the visual stimulus bejond the beginning or end of the eye movement. This is a temporal visual manipulation. and any corollar discharge should be equally active in both the case where the smear is seen and the case where it is not Finally, a stationary blank flash preceded or followed by a clear image is obscured by the clear image and this occurs entirely independently of any eye move* ment at all. While these points do not argue aganst 
the concept of a corollary discharge (which might prevent the world from appearing to move, as Helmholtz suggested). they indicate that such a discharge is unnecessary to account for the lack of visual perception during eye movements in a normal contoured visual environment.

Both Dodge $(1900,1905)$ and Woodworth (1906) emphasized the blurred or "fused" image which must occur during a saccade, but much of the subsequent experimental work has deliberately eliminated the blurred image. In order to reveal the existence of a central inhibitory mechanism which might be obscured by the effect of a smeared image. Volkmann (1962) measured the threshold rise of a small test target. briefly pulsed and therefore sharp. added to a background before, during, or after a saccade. This experiment, and subsequent ones (see Matin, 1974. for review) have generally revealed a small elevation in the threshold for detecting a flashed target. This change in threshold is so slight that neither Dodge nor the present authors would have been able to detect it in their experiments, and it certainly would not account for our failure to see the grey-out of an eye movement. as indicated by Volkmann (1962): "The present results suggest some characteristics of the magnitude and the time course of the inhibitory effect. They suggest, first, that the magnitude of the effect is not large. The moving eye required a stimulus flash of about $0.5 \mathrm{log}$ unit greater luminance than did the fixating eye in order to detect the flash or to recognize words when they were presented by the flash. The finest gratings which could be resolved by the moving eye were about 1.4 times as coarse as the finest ones resolved by the fixating eye. When one considers the ranges of luminance and stimulus-detail which we encounter every day in the visual scene, differences of these magnitudes are not impressive. It is doubtful whether central inhibition alone can account for the phenomenal 'continued clear vision' which occurs under a wide variety of circumstances. Instead. the fact that we do not notice stimuli which arrive during eye movements under normal circumstances must be attributed largely to retinal smear, which serves to decrease the photochemical effect of stimulation."

We have in fact found that this comparison of retinal smear during the eye movement and the clear vision after the eye movement is essential to understanding our failure to see the grey-out of a saccade. It should be noted, however, that other mechanisms must account for our lack of vision during micro-saccades since the velocity of these saccades is not high enough to produce a retinal smear.

The slight rise in threshold for detection of brief test stimuli presented during eye movements has been referred to as "saccadic suppression" (as cited by Zuber and Stark. 1966). The saccadic suppression studies leave open the question of whether the perceived luminance of the background and the test spot are decreased, or whether the background appearance remains constant and the contrast sensitivity of the visual system is decreased. Volkmann. Riggs. Moore and White (1978) have made an important contribution by using a grating of variable contrast as the test stimulus, thus measuring contrast sensitivity during saccades. The maximum reduction in contrast senstivity found by Volkmann et al. (1978) is $0.5 \mathrm{log}$ units. For the medium range of spatial frequencies (around $3 \mathrm{c} / \mathrm{deg}$ ) the visual system has a contrast sensitivity as low as $0.5^{\%}$.
Thus, the total range of contrast sensitivity for these important spatial frequencies is $200: 1$ or $2.3 \mathrm{log}$ units range. It is clear that a change of only $0.5 \mathrm{log}$ units lasting for less than $100 \mathrm{msec}$ (Volkmann et al. 1978) is a minor one. Even if the decrease in contrast sensitivity during a saccade were much greater it would be of little importance. Consider an image either completely greved-out due to its high velocity on the retina. or an image partly smeared and therefore of low contrast. Clearly if one decreases the contrast sensitivity of the eye it will do nothing to an image which is already of zero contrast. and little to an image of low contrast.

Until Volkmann et al. (1978) established that the change in sensitivity of the eye is in the domain of contrast sensitivity. much of the literature suggested that this change of sensitivity took the form of dimming the visual scene during a saccade. such as indeed happens physically during blinks. But we can be fully aware of every blink we make because of the dramatic decrease in retinal illumination. This experience is quite unlike that found with saccades: if there was a substantial decrease in light sensitivity during a saccade it would be perceived as a dimming of the visual world. It is thus vitally important that little or no gain change occurs in the visual system during a saccade. The elimination of the grey-out by the clear image before and after the eve movement avoids any such change in the gain of the visual system: there is an omission of the perception of the grey-out: a saccadic omission. not a slight increase in threshold; a saccadic suppression.

\section{Visual masking}

This saccadic omission is of course a visual masking phenomenon in that one visual stimulus obscures another one. Backward masking or meta-contrast has been suggested previously as the mechanism producing the elevated detection threshold called saccadic suppression (Alpern, 1969; Mackay. 1970; Grusser. 1972; Breitmeyer and Ganz, 1976). Mackay (1970) demonstrated that such an increase in threshold occurs even if remote contours in the background are moved in the absence of any eye movement. Subsequent experiments have confirmed and extended this effect (Mitrani. Meteeff and Yakimoff, 1971; Brooks and Fuchs. 1975; Mateeff. Yakimoff and Mitrani, 1976) although the experiments do not exclude some residual effect of eye movement (Riggs, Merton and Morton, 1974). These masking effects. like saccadic suppression. have generally been only about $0.5 \mathrm{log}$ units of threshold elevation. However. where a patterned background has been used (Brooks and Fuchs. 1975) the effect is substantially greater.

The effect of the stimulus after the eye movement on the smeared image during the eye movement was first studied by Matin et al., (1972). In these experiments a slit of light against a black background was illuminated during a $4^{\circ}$ long saccade. As the duration of the illumination extended $100 \mathrm{msec}$ beyond the end of the saccade the stimulus was seen as.it was by the fixating eye; no smear was then reported. Matin et al. (1972) suggested that a "temporally backward and spatially lateral inhibition (metacontrast) occurs when the duration of the flash is long enough to permit sufficient temporal integration of illumination in the period after the saccade to develop a 'mask'." They went on to suggest that for saccades occurring in more complex environments masking might be more effective and that forward as well as backward masking might occur. The present experiments show that the masking effect is the primary factor in a 
complex environment as long as the image during the saccade is smeared or greyed-out: a clear image during a saccade is easily percerved. In addition we have found that forward masking is just as effective as backward masking.

In summary. our view of the importance of the smeared image during an eye movement and the action of the clear image before and after in obscuring the smear represents a return to the vlews of Dodge in 1900: "Moreover, it must be remembered that the duration of the eye movements is very short, for movements of $10^{\circ}$ certainly not more than $60 \mathrm{msec}$. and possibly less than half that. It will be seen at once that the equalizing stimulation during the movement of the eyes scarcely covers the duration of the most pronounced after-effect of the intense stimulation at the preceding fixation pause: while the interference of the intenser stimulation of the succeeding fixation would make the faint stimulation hardly perceptible. even if it had no after-effect of the previous fixation, and no central inhibition to orercome in establishing itself." However. Dodge later went on (1905) to suggest that attention was also a significant factor in the lack of perception during eve movement, an addition we think is not necessary.

\section{Neurophysiological correlates}

The salient point of our experiments is that no oculomotor or proprioceptive signal is needed to eliminate perception during saccadic eye, movements. We would expect. instead, an interaction within the visual system itself. Wurtz (1969 a,b) first investigated the response of neurons in the monkey visual system (presumably similar to that in man) to visual stumuli during eye movements. He found that cells in visual cortex (area 17 or striate cortex) responded to retinal image movement in the same manner whether the movement was produced by movement of the eye or the stimulus. and thus there was no evidence of a corollary discharge. Buittner and Fuchs (1973) also failed to find a significant modulation of activity of lateral geniculate cells in the monkey in association with saccades made in the dark. These neurophysiological results are consistent with our psychophysical conclusion that a corollary discharge need not be involved in saccadic omission. However, Wurtz (1969b) also found that many neurons did discharge during an eye movement. But since, in these experiments, the stimulus was present on the receptive field of the cell during the eye movement but not before or after the eye movement. these results are more comparable to our psychophysical experiments in which a smear was seen during the eye movement-presumably due to activation of an assembly of similar receptive fields. In subsequent experiments where the stimulus was on the receptive field of the cell before and after the eye movement, the cell did not respond to the stimulus during the eye movement (Judge and Wurtz. 1977). These results are comparable to the present psychophysical experiments when a clear stationary scene was present before and after the eye movement and the grey-out was not seen.

Several recent studies have shown a slight modulation of activity of cells in the geniculo-striate pathway of monkeys (Duffy and Birchfield. 1975: Bartlett. Doty. Lee and Sakakura. 1976). This indication of an extra-visual input is not in conflict with the previous results of Wurtz (1969a.b) since to see this input requires averaging over many trials whereas the previous results rested on a trial by trial analysis. That non-visual effects can be seen on such a trial-by-trial basis is demonstrated in the monkey superior colliculus (Robinson and Wurtz. 1976) where cells receive an extra-retinal input that eliminates the response of these cells to stimulus movement resulting from an eye movement (although these cells probably play no role in saccadic suppression). The slight physiological effects seen with averaging at the striate cortex are reminiscent of the slight $0.5 \mathrm{log}$ unit elevation of visual threshold of saccadic suppression. Taking the neurophysiological and psychophysical experiments together, our hypothesis would be that the inability to see the grey-out during a saccade (saccadic omission) results from interaction within the visual system and depends on no extra-visual input; this is comparable, at a psychophysical level, to the results at a physiological level (Wurtz. 1969a.b). The slight elevation in detection threshold (saccadic suppression) might be comparable to the slight modulation of cortucal activity which requires averaging over several trials to be clearly established (Duffy and Birchfield. 1975: Bartlett et al., 1976).

\section{REFERENCES}

Alpern M. (1969) Saccadic eye movements. In The Eye. Vol. 3, Movement of the Eyes (edited by Davson $\mathrm{H}$ ). pp. 98-99. Academic Press. New York.

Bartlett J R., Doty R. W. Sr, Lee B. B. and Sakakura H. (1976) Influence of saccadic eye movements on genıculostriate excitability in normal monkeys Expl Bram Res. 25, 487-509.

Brestmeyer B G. and Ganz L. (1976) Implications of sustained and transient channels for theories of visual pattern masking. saccadic suppression. and information processing. Psychol Ret. 83, $1-36$.

Brooks B. A. and Fuchs A. F. (1975) Influence of stımulus parameters on visual sensitivity during saccadic eye movements. Viston Res. 15. 1389-1398

Büttner U. and Fuchs A. F. (1973) Influence of saccadtc eye movements on unit actuvity in simian lateral geniculate and pregeniculate nucler. $J$. Veurophysiol. 36. $127-141$.

Corfield R. Frosdick J. P and Campbell F W (1978) Grey-out elimination. The roles of spatial waveform. frequency and phase. Vision Res. This issue. pp. 1305-1311.

Dodge R. (1900) Visual perception durng eve movement Psichol. Rev 7, 454-465.

Dodge R. (1905) The lllusion of clear vision during eve movement. Psychol. Bull. 3. 193-199.

Duffy F. H and Birchfield L. (1975) Eye movement-related inhibition of primate visual neurons. Bram Res. 89. 12!-132.

Grusser O. J. (1972) Metacontrast and the perception of the visual world Pfliggers Arch-European $J$. Phystol 332. R98

Helmholtz H. von (1910) Treatise on Physiological Optics. Vol. III (edited and translated from 3rd edn by Southall J. P. C.). Optical Society of America. Menasha. WI.

Holst E. von (1954) Relations between the central nervous system and the peripheral organs. Br. J. Anumal Behat. 2. $89-84$

Holt E. B. (1903) Eye-movement and central anaesthesia Harcard Psychol. Studies 1, 3-45.

Judge S. J. and Wurtz R. H. (1977) Visual responses during saccadic eve movement: Visual masking in striate cortex. Veuroscl. 4bstr. 3, 564 
Mackay D. M. (1970) Elevation of visual threshold by displacement of retinal image. Nature 225, 90-92.

Mateeff S.. Yakimoff N. and Mitrani L. (1976) Some characteristics of the visual masking by moving contours. Vision Res. 16. 489-492.

Matin E. (1974) Saccadic suppression: A review and an analysis. Psychol. Bull. 81. 899-917.

Matin E., Clymer A. B. and Matin L. (1972) Metacontrast and saccadic suppression. Science, 178. 170-181.

Mitrani L., Mateeff S. T. and Yakimoff N. (1971) Is saccadic suppression really saccadic? Vision Res. 11, $1157-1161$.

Riggs L. A., Merton P. A. and Morton H. B. (1974) Suppression of visual phosphenes during saccadic eye movements. Vision Res. 14, 997-1011.

Robinson D. A. (1964) The mechanics of human saccadic eye movement. J. Physiol. 174, 245-264.

Robinson D. L. and Wurtz R. H. (1976) Use of an extraretinal signal by monkey superior colliculus neurons to distinguish real from self-induced stimulus movement. J. Neurophysiol. 39, 852-870.

Sperry R. W. (1950) Neural basis of the spontaneous optokinetic response produced by visual inversion. $J$. comp. physiol. Psychol. 43, 482-489.

Volkmann F. C. (1962) Vision during voluntary saccadic eye movements. J. opt. Soc. Am. 52. 571-578.

Volkmann F. C., Riggs L. A.. Moore R. K. and White K. D. (1978) Central and peripheral determinants of saccadic suppression. In Eye Movements and the Higher Psychological Functions (edited by Senders J. W.. Fisher D. F. and Monty R. A.). Hillsdale. N.J. In press.

Westheimer G. (1954) Mechanism of saccadic eve movements. Archs Ophthal. 52, 710-724.

Woodworth R. (1906) Vision and localization during eye movements. Psychol. Bull. 3, 68-70.

Wurtz R. H. (1969a) Response of striate cortex neurons to stimuli during rapid eye movements in the monkey. J. Neurophysiol. 32, 975-986.

Wurtz R. H. (1969b) Comparison of effects of eye movements and stimulus movements on striate cortex neurons of the monkey. J. Neurophysiol. 32. 987-994.

Zuber B. L. and Stark L. (1966) Saccadic suppression: Elevation of visual threshold associated with saccadic eye movements. Expl. Neurol. 16. 65-97. 